\title{
Laser and Fiber Electronics Group
}

\author{
Jarosław Sotor, Krzysztof Abramski, Arkadiusz Antończak, Grzegorz Soboń, Paweł Kaczmarek, Adam Wąż, \\ Grzegorz Dudzik, Karol Krzempek, Bogusz Stępak, Łukasz Sterczewski, Dorota Stachowiak, Jakub Boguslawski, \\ Maciej Kowalczyk, Aleksander Głuszek, Arkadiusz Hudzikowski, Maria Pawliszewska, Olga Drożdżowska, \\ Zbigniew Łaszczych, Michał Wójcik, Katarzyna Łęcka, Paulina Dzienny, Jarosław Szumega, Dorota Tomaszewska, \\ Michał Porębski \\ Wroclaw University of Science and Technology, Faculty of Electronics, Department of Field Theory, Electronic \\ Circuits and Optoelectronics
}

Received June 08, 2019; accepted June 24, 2019; published June 30, 2019

\begin{abstract}
The Laser \& Fiber Electronics Group (LFEG) constitutes a team of young, skilled and ambitious researchers, doctoral candidates and students. The Group possesses great experience in applied optoelectronics and laser technology. Currently, it conducts research in several areas, mostly focusing on: ultrashort laser pulse generation using novel materials, development of pulsed fiber laser sources ranging from visible to mid-infrared, development of compact mid-infrared optical frequency combs, laser spectroscopy techniques, laser vibrometry, solid-state lasers, advanced analog and digital electronics, and investigations of light-matter interaction.
\end{abstract}

The Laser \& Fiber Electronics Group (LFEG) constitutes a team of young, skilled and ambitious researchers, doctoral candidates and students. The Group possesses great experience in applied optoelectronics and laser technology. Currently, it conducts research in several areas, mostly focusing on: ultrashort laser pulse generation using novel materials, development of pulsed fiber laser sources ranging from visible to mid-infrared, development of compact mid-infrared optical frequency combs, laser spectroscopy techniques, laser vibrometry, solid-state lasers, advanced analog and digital electronics, and investigations of light-matter interaction. The Group has a well-established position in the research community and the obtained results are worldwide recognized. In recent 10 years, the research results were published in $>90$ JCRindexed journals with IF ranging from 2.5 to 13.3 , which were already cited $>2000$ times. The Group continuously carries out research grants funded by the Polish agencies: National Science Centre (NCN), National Centre for Research and Development (NCBiR), Foundation for Polish Science (FNP) and the Polish Ministry of Science and Higher Education (MNiSW). The total amount of funding received within research grants in recent 10 years exceeds 40M PLN. The Group's ambition is to constantly improve the research quality and transfer the results to the industry. The Group has developed several prototypes of various laser-based and optoelectronic devices. Currently the Group participates in the Roadmap of Polish research infrastructure project: National Laboratory of Photonic and Quantum Technology (NLPQT), a consortium uniting the leading Polish institutions in the area of photonics and quantum technologies.

Currently the Group consists of 1 full professor, 3 associate professors with habilitation, $8 \mathrm{PhD}$ 's, $10 \mathrm{PhD}$ students and 4 undergraduate students. Each year at least 1-2 new young researchers join the team. The Group also hosts researchers from other institutions within a broad national and international collaboration. The competence of the group members covers the following areas: fundamental research in the field of photonics and optoelectronics; practical realization of advanced mechanical and electrical systems and sub-systems; development of software solutions for research purposes; integration of sub-systems and development of prototypes; seeking for applications of basic research. In recent years, the Group focused on gathering and extending the knowledge in the area of photonics, which is an extremely important branch of science. These activities ideally match with the European Commission strategies and programs, e.g. Photonics 21. It is worth noting that photonics is also one of the national intelligent specializations in Poland (so called "KIS").

The Laser \& Fiber Electronics Group currently conducts research within three laboratories:

\section{1) Lasers and optical amplifiers laboratory}

The laboratory carries out research in the following areas: - generation of ultrashort laser pulses - one of the main scientific interests of the Group. The goal is to develop novel techniques of generating high-energy and high-peak power ultrashort pulses, outperforming the current state-ofart constructions. Within this topic, the Group has developed a prototype of a graphene-based femtosecond fiber laser, and conducts research on other novel materials, e.g. black phosphorus, antimony telluride, carbon nanotubes, etc. The Group is developing mode-locked fiber 
lasers based on $\mathrm{Yb}$, Er, Tm and Ho-doped gain media, covering the spectral range from 1 to $2.2 \mu \mathrm{m}$ using different saturable absorbers.

- high power fiber amplifiers operating in pulsed and CW regimes at 1064, 1550 and $2000 \mathrm{~nm}$ wavelengths - one of the main interests is the development of multi-channel coherently combined sources at $1064 \mathrm{~nm}$ for high-power output. Within this task, amplifiers, seed sources and phase-locking algorithms are developed.

- Mode-locked solid-state lasers - the research focuses on generation of ultrashort pulses from solid-state lasers operating at $1030 \mathrm{~nm}$ (Yb-doped gain media) or $2000 \mathrm{~nm}$ (Tm-doped gain media) with the use of novel nanomaterials as saturable absorbers. The research is carried out in the frame of the SONATA BIS programme funded by NCN.

- optical frequency combs in the mid-infrared generation and spectral conversion of frequency combs by means of nonlinear techniques (e.g. OPO, DFG, supercontinuum generation in photonics crystal fibers) and applications of frequency combs in laser spectroscopy. Recently, the Group has developed a stabilized Er-doped fiber laser comb and fully fiberized sources of mid-infrared combs tunable from 6 to $9 \mu \mathrm{m}$. The research is carried out in the frame of First TEAM programme funded by FNP.
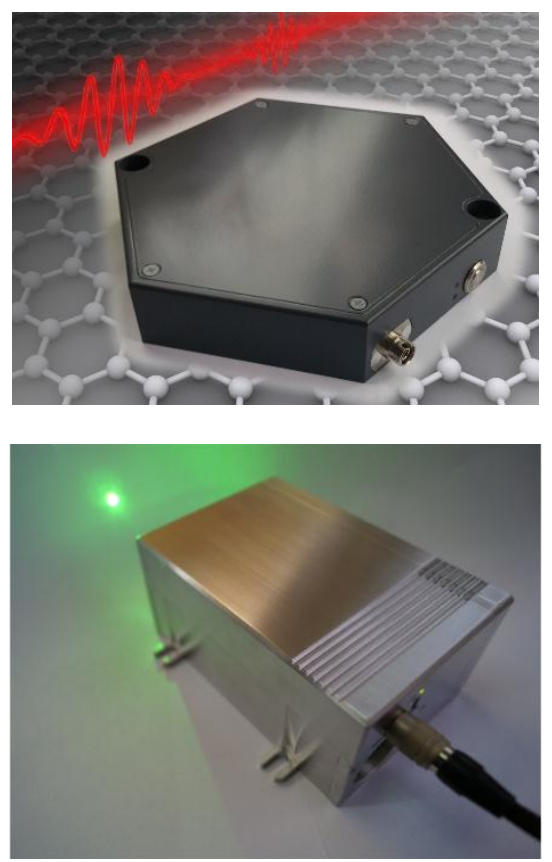

Fig. 1. Prototype of a femtosecond laser with graphene-based saturable absorber (top), prototype of a single-frequency green laser - project for the European Space Agency (bottom).

\section{2) Laser spectroscopy and applications laboratory}

The laboratory is based on the Group's great experience in stabilization and frequency control of many different types of lasers (gas, semiconductor, solid-state microlasers) and fruitful collaboration in two world-leading spectroscopy laboratories: Princeton University (Prof. Gerard Wysocki, former student of the Group) and Rice University Houston (Prof. Frank Tittel, and a joint PhD student Rafal Lewicki). Within this laboratory, the Group develops novel spectroscopic techniques based on original and patented designs of diode-pumped solid-state lasers (DPSSL). These gas sensors are characterized by exceptional simplicity, compactness and detection sensitivity, and are based on inter alia so called phototermal effect. The recent research efforts are devoted to new sensing techniques using hollow-core photonic crystal fibers (HC-PCFs). Such configuration also enables further miniaturization of the sensor and improving the detection limit. The research on compact multipass cells is another part of work aimed at developing effective gas sensors fitting the trend of miniaturization.

\section{3) Laser microprocessing laboratory}

In the past decade the Group developed many important technologies connected with laser micro-processing of different materials, for example:

- fabrication and prototyping of micro-optical and electrical structures (micro-antennas, RFID, MEMS, MOEMS),

- fabrication and characterization of medical implants (e.g. bio-degradable coronary stents),

- laser-based metallization of AlN ceramics and development of metamaterials structures using this technology,

- fabrication of hydrophobic surfaces based on microstructurization (e.g. laser induced periodic surface structures - LIPSS technique),

- development of a metal color marking technology (awarded with Gold Medal Brussels Innova 2012),

- development of laser-induced anti-corrosion coatings for austenitic stainless steel,

- laser modification of polymers (and analysis of their physicochemical properties). 
One of the Group's specialties is also spectroscopy and material analysis with the use of laser-induced breakdown spectroscopy (LIBS). The possessed equipment and human potential make this laboratory the best laser micromachining laboratory in Poland. It is equipped with several workstations based on pulsed lasers: CO2 (10.6 $\mu \mathrm{m})$, excimer ArF (193 nm, $10 \mathrm{~ns}), \mathrm{Nd}$ :YAG lasers (1064 $\mathrm{nm}, 532 \mathrm{~nm}, 10 \mathrm{~ns}$ ), and Ytterbium-doped fiber laser (1030 $\mathrm{nm}$, tunable pulses from $200 \mathrm{fs}$ to ps, TRUMPF TruMicro). The last system, obtained within a fruitful collaboration with TRUMPF Laser GmbH company, opens a new research area for investigations of light-matter interactions. The laboratory participated in several research projects devoted to micromachining (among them funded from EU/POIG).

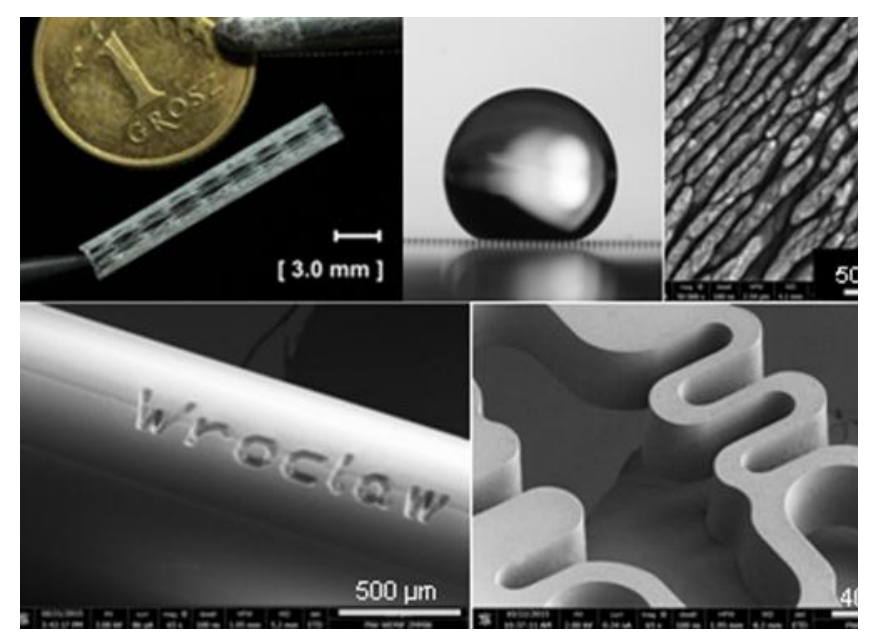

Fig. 2. Examples of objects made in the Laser Micrprocessing Laboratory (biodegradable stents, Fresnel microlenses, LaB-on Chip structures). 